\title{
World and mind, information and semantic content
}

\author{
Manuel Liz
}

Faculty of Philosophy; Dept. of History and Philosophy of Science, Education and Language; University of La Laguna, Canary Islands, Spain; E-mail: manuliz@ull.es

\begin{abstract}
Many times, the notion of information is used in such a way that the following two theses are suggested: 1) that the world might be no more than information, and 2) that our minds might be no more that information. This paper rejects both theses. In relation to that, I will argue for the need to take into account non-informational aspects of reality that are epistemically accessible. Only that way, we could deal with the problem of selecting a determinate semantic content and with the problem of error. The two more common strategies to deal with these problems appeal to some primitive "referential capacities" or to some special kinds of (natural) "functions". We propose another strategy based on very simple processes of signalization. With the help of that strategy, we offer a new way of defining semantic content.
\end{abstract}

Keywords: Information, physical properties, semantic content, mind, signals, referential capacities, functions, noninformational access.

Acknowledgement: This paper has been elaborated in the context of the Research Projects HUM2005-03848 and FF12008-01205 (Spain). Some of the material has been discussed in the I International Meeting of Experts in Theories of Information, held in Leon, November of 2008. I want to thank to that audience for all kinds of helpful discussions and perceptive comments.

$\mathbf{n}$ this paper, I want to discuss a number of problems and proposals having to do with the notion of information. All of them are largely independent on the particular concept of information that is adopted. But, both the problems and the proposals will be very relevant in relation to the definition of the peculiar semantic contents that can be involved in any informational process.

In the first section, I will try to make explicit the general conceptual framework in which our analyses will take place. And I will pose three very important ontological problems. Very briefly, the problems in question will be: 1) might the world be no more than information, 2) might the mind be no more than information, and 3) is there any relevant relationship between the two previous problems. In the three next sections, I try to offer some answers to those questions. I will argue that the world cannot be constituted only by information, and that our minds cannot be constituted only by information either.
Moreover, even assuming that some sort of propositional content can be defined in informational terms, I will argue that for our minds to have the kind of highly selective propositional contents they are able to have, a kind of propositional content that also can be erroneously exemplified, our minds have to be able to have some sort of direct epistemic access to the non informational aspects of the world. By the way, I will introduce some new ideas about how those selective and potentially erroneous propositional contents could be achieved. And I will do it without appealing to the existence of any sort of irreducible "referential capacities" and without any appeal to the notion of "functions" in the usual sense of the term.

\section{A general framework for the notion of information}

Even though the bearers of information are always physical entities having physical 
properties, information itself maintains much less direct relationships with the physical world. Information does not determine any particular physical properties. This introduces a very important indetermination. But there is also another not less important indetermination coming this time from the semantic contents that can be associated with certain information. Information can be measured. We can measure quantities of information. However, we have to distinguish between quantities of information and the semantic content associated with certain information. And the crucial point is that semantic contents are not only quantities of information.

\subsection{Information and physical properties}

There is no information without something able to bear that information. Hence, some bearers of information need to be introduced. And such bearers of information always are physical entities (objects, events, states of affairs, etc.) able of having physical properties of a "first order" kind. In contrast, information itself would be a "second order" property. It is the having other properties, or relations, of a first order kind what makes that something is able of having certain information. We can consider information as a physical property, but in that case it has to be clear that we would be faced with second order physical properties.

Also, it is possible, and in fact very usual, to characterize information as a "functional" property. Every functional property would be a second order property. But not every second order property would be a functional one. Being a functional property does not only requires that it is definable through the quantification, in the logical sense, over other first order properties, but that those properties satisfy a certain sort of relational structure.

In any case, the relationships between information --informational properties-- and first order physical properties are not direct. The same point can be made using the notion of supervenience. Information would supervene on (other) physical properties in the sense that if some physical properties are had, including here relational physical properties, then necessarily certain information is had. In other words, variations concerning information always have to entail variations in those physical properties. Information is determined by physical properties. However, things are not so in the reversed way. Variations concerning those physical properties do not have to entail variations in information. Information does not determine any particular, constitutive or relational, physical property for the bearers of that information.

As a consequence, the relationships between information and causality cannot be direct either. Even though the bearers of information are causal effects, and they can have causal effects, in relation to other objects, events, states of affairs, etc., the having certain information does not determine any particular causal relation. The having certain information does not determine that the bearers of that information are causally produced in any particular way. And it does not determine either that they have some particular causal effects.

\subsection{Information and "form"}

Many of the above features are closely connected with the meaning of the linguistic root "form". The "form" of an object, i.e., its shape -including here, in that generic sense of "form", things like the size of the object-, is also a second order property. It can be considered a physical property. And it supervenes on (other) first order physical properties. The "form" of an object is determined by them. However, the fact that an object has a certain "form" -triangular, squared, ovoid, large, etc.- does not determine its physical properties, constitutive or relational. And it does not determine either the causal relations in which an object with that "form" is involved.

It is convenient to note that all the above mentioned facts are perfectly compatible with the fact that objects with certain "form" have some constitutive or relational properties. It is also compatible with the fact that they are causal effects of other things, and can cause other things. And it is also compatible with the fact that the having a certain "form" is able of bounding, even in very relevant ways, both 
the kinds of things that are able to cause them and the kinds of things that can be causal effects of them.

\subsection{Quantities of information}

Information can be measured. Information is measured in quantities of information -bits, entropy, qubits, etc.--. The quantity of information sizes the capacity of having information.

In that sense, information would be a mathematically quantifiable property. The quantification can be made in relation to certain statistical probabilities attributed to the bearers of information, in relation to the degree of relative disorder of those bearers, considered as thermodynamic systems, in relation to the probabilities associated with some of the quantum states of the bearers which could yield some sort of values, other sort of values and, also, an entanglement of both sorts of values--, etc. What would be obtained is, respectively, 1) a mathematical concept of information based on statistical probability, 2) a physical concept of information based on entropy, and 3) the quite recent concept of quantum information. (In other section we will address the use of the notion of information in biology)

As we have said, to measure quantities of information is to measure a peculiar sort of capacity. It is like to measure the capacity of a certain glass, of certain "form" -shape, size, etc.-in order to contain a determinate volume of liquid. The glass could contain $1 / 2$ litre, 1 litre, 5 litres, etc. To know that, however, would be to know nothing determinate about the constitutive or relational physical properties of the glass. And it would be to know nothing determinate about its causal properties either. Moreover, we would know nothing either about what kind of liquid the glass contains -if it contains any.

\subsection{The semantic content of information}

An answer to the last question (What kind of liquid does the glass contain?), in the case of information, would be obtained when we ask about the concrete and particular information which is contained in the bearer - what the bearer is "saying"--. In other words, information also can have a peculiar semantic content. It would be a very interesting question to ask whether it could make sense to speak properly about information without any semantic content, but we do not need to face that question here. It would be enough to assume that information can have semantic contents.

As very simple cases of semantic content, we can think on propositionally articulated semantic contents -i.e., propositions- of the sort that $a$ is $F$. We would describe the semantic content of an information saying, for instance, that it consists in that $a$ is $F$. To describe semantic contents is like saying what is contained in a certain glass: $1 / 2$ litre of water, 1 litre of milk, 5 litres of wine, etc.

First order physical properties of the bearers of information can be understood as the non-informational properties that are mentioned when reality is described from the point of view of our more basic sciences ${ }^{1}$. And, according to what we are saying, semantic content would be the sort of propositionally articulated concrete information that can be cognitively relevant from a psychological perspective ${ }^{2}$.

\subsection{Three problems}

The quantity of information that a certain bearer can contain does not determine the physical properties of the bearer -constitutive, relational, causal, etc.--. And that quantity of information does not determine either the semantic content of the information, the concrete information contained in such a bearer -what that bearer is "saying". The capacity for containing certain "quantity of information" is compatible with a great variety

\footnotetext{
${ }^{1}$ In fact, the notion of information might be present in our more basic sciences. But a consequence of what we will argue in next sections is that science, and a fortiori basic sciences, cannot describe the world mentioning only informational properties. And this would be true even in relation to the ways quantum physics can be interpreted.

${ }^{2}$ Briefly, propositions would be those kinds of entities able of being the objects of our propositional attitudes beliefs, desires, etc.--, and those kinds of entities able of maintaining inferential relationships -deductive, inductive, etc.
} 
of propositionally articulated semantic contents.

We can say that the metaphysical status of information is not very well defined. Information appears to occupy some place between, on the one side, the physical world constituted by first order physical properties and relations and, on the other side, the world of meanings, contents and propositions psychologically relevant --at least for the cognitive part of our minds.

The peculiar metaphysical status of information is the source of many problems. Here, I want to discuss three of them. They are the following ones:

1) Might the world be no more than information?

2) Might the mind be no more than information?

3) Is there any relevant relationship between problems 1 and 2 ?

\section{Might the world be no more than information?}

We will argue for a negative answer to that question. The world cannot be only information. Moreover, the world that can be epistemically accessible cannot be either only information. In particular, not all knowledge of the world can be propositionally encapsulated in the form of semantic contents of the sort that $a$ is $F$. We will argue that in order to be possible any knowledge of the world articulated through propositional contents, it has to be also possible to know the world, or to access to it, in other ways.

\subsection{Two senses of the question}

The question above posed (Might the world be no more that information?) can have two main senses, one of them strongly ontological and the other one much more epistemological.

The idea that, in the end, the world might be only information, in a strong ontological sense, is nowadays present in some trends of thought. But, according to what we have said about the bearers of information, and about the relation of supervenience of information on (other) physical properties, strictly the world cannot be only information. It would be like stating that there can exist the capacity of containing a volume of, let us say, $1 / 2$ litre, 1 litre, 5 litres, etc., without existing any sort of container at all.

Perhaps, the idea that the world might be only information did not have a purely ontological meaning but an epistemological one. Then, by "the world" we would have to understand "the world epistemically accessible". And what is stated would be that, in the end, we do not have access to the world but through information. Let us analyze more closely that suggestion.

\subsection{Having access to the world only through information}

But, "having access to the world only through information" would have to have here an interpretation full of very diverse semantic contents. The accessible world would become something implausibly narrow if we only could know about it quantities of information, or may be relations among quantities of information. We never could state about the world something like that $a$ is $F$, being $a$ and $F$ not only quantities of information. However, the rule is to make very often statements of that sort. And unless it is showed how they could be translated, or made equivalent, to other statements involving only quantities of information, and it is very doubtful that it can be made, we would have to conclude that the real problem is to explain how we can have the great variety of semantic contents that in fact we are able to have.

Hence, "having access to the world only through information" has to mean "having access to the world only through some semantic contents propositionally articulated, i.e., semantic contents consisting in things like that $a$ is $F$, being $a$ and $F$ not only quantities of information. But, how those semantic contents are originated? And how to explain the ways in which we get to access to the world through those semantic contents?

\subsection{Explaining semantic contents}

The explanation of semantic contents really is a complicate matter. But a very influential approach has been the one proposed by Fred 
Dretske in the last years ${ }^{3}$. We will introduce briefly that approach. And it will serve us to identify three big problems. Dretske defines the semantic content of a particular signal in the following way:

\section{A signal s, registered in a signal receiver $r$, and coming from a certain source $a$, has the semantic content that a is $F$ iff the conditional probability that the source $a$ is $F$, given the signal $s$, and given some previous knowledge $K$ that $r$ has about the possibilities of being $F$ of $a$, is 1. (Ibid)}

For example, if given the signal $\mathrm{s}$, the probability that $a$ is $F$ or $\mathrm{G}$ or $\mathrm{H}$ is 1 , and $\mathrm{K}$ consists in that a cannot be $\mathrm{H}$, then the signal $s$ will have, for $r$, the disjunctive semantic content that $\mathrm{a}$ is $\mathrm{F}$ or $\mathrm{G}$. If the receiver $r$ does not have any knowledge $K$ about the possibilities of the source $a$, then the signal $s$ will have the semantic content consisting in that $a$ is in a certain way if, given $\mathrm{s}$, that $\mathrm{a}$ is that way has a probability of 1 .

That simplification of Dretske's position would serve to make explicit three big problems that any explanation of semantic content would have to face. They are the following:

1) The problem of the need to take into account some previous knowledge $K$ :

The need to take into account $K$ presupposes a set of previous semantic contents that would have to be explained. This could lead to situations of regress or to situations of circularity. Of course, this problem would not be a hard one if we can assume that in the last term it would be possible to avoid $K$. We will take it for granted in our discussion. In any case, and even though such assumption may be seem as plausible, we would be faced with the next two problems. ${ }^{4}$

\footnotetext{
${ }^{3}$ Mainly, in Dretske, F. (1981). Adopting the general framework of Shannon and Weaver, Dretske tries to make precise the notion of semantic content of a particular signal -"cognitive content" in his terminology.

4 The need to take into account some previous knowledge in order to explain semantic content is a general problem for the selection of any semantic content: mental contents, linguistic meanings, the intentional contents of actions, the normative contents of rules, etc.
}

2) The problem of getting selective semantic contents:

According to the definition of semantic content above introduced, the signal $s$ will have as semantic content every feature had by a with a probability of 1 given $s-$ we assume that any reference to $K$ could be eliminated--. However, $a$ itself can register signals coming from other sources. Moreover, these other sources can register signals coming from other ones. In the last term, the signal $s$ could have in its semantic content all the features of those other sources that have probability 1 given $s$. This makes of the semantic content of any signal something quite broad. There would be a kind of explosion in the semantic content of every signal -and everything can be a signal ${ }^{5}$. Hence, in order to select the relevant semantic content of a signal it would be required some non-regressive and noncircular procedure to narrow that semantic content.

3) The problem of error:

According to the above definition of semantic content for a particular signal, error would not be possible. If a signal has the semantic content that $a$ is $F$, then necessarily $a$ is $F$. This generates a problem because, unless from an intuitive point of view, there would have to be room for semantic contents erroneously exemplified. The semantic contents of many of our beliefs, for instance, seem to be semantic contents that can be erroneously exemplified. Sometimes this problem is interpreted as deriving from Dretske's requirement that the probability that $a$ is $F$, given $s$-we follow assuming an elimination of $K--$, is 1 . Indeed, that requirement entails a great amount of idealization about informational channels. In fact, informational channels always make that some information is modified or lost. But the real problem is not here. The crucial problem is that the correctness of semantic content is too much closely

\footnotetext{
5 Not only can everything be a signal. Everything would have as semantic content information over quite very large parts of the whole history of the universe, going back to the very Big-Bang.
} 
linked to the exemplification of that semantic content. If in order to have the semantic content that $a$ is $F$ some other probability $p$ less than 1 were required, and no other modification in the above definition of semantic content were introduced, then exactly in the same degree that semantic content would be correct. In other words, that semantic content would have a probability of error of $1-p$, which is exactly the probability that $s$ does not have the semantic content that $a$ is $F$. What is required is more distance between, on the one hand, the having the semantic content that $a$ is $F$ and, on the other hand, the fact that it is correct to have that content. Moreover, it is needed something able of explaining how a semantic content can be non erroneously exemplified with less probability than the probability of exemplifying that content. And it is needed to do it without making reference to semantic contents with respect to which the problem of error remains open. That would be regressive. Moreover, it is needed to do it without introducing semantic contents which are merely postulated as capable of being erroneously exemplified. That would be circular.

The last two problems have been recently discussed with detail by many authors ${ }^{6}$. They are also very old problems ${ }^{7}$. And they would be problems that have to be faced by any explanation of how a signal can have the particular semantic contents that it is intended to have. Moreover, they would be crucial problems for the claim that the only knowledge we can have about the world is the kind of knowledge encapsulated in semantic contents of the sort that $a$ is $F$.

Let us resume our results. Does the world consist only in information? Our answer was negative. The bearers of information cannot be themselves only information. Does our access to the world consist only in information? Our answer was also negative.

\footnotetext{
${ }^{6}$ Among them, we would have to mention Millikan, $\mathrm{R}$. (1984) and Fodor, J. (1990).

7 In particular, the problem of error is discussed in deep by Plato in his Cratylus.
}

We know about the world more than quantities of information. Is the world only known through propositionally articulated semantic contents of the sort that $a$ is $F$, being $a$ and $F$ not only quantities of information? Faced with the problems we have presented, an affirmative answer would have to explain in a non-regressive and non-circular way how can the intended semantic contents exist.

\subsubsection{Knowing the world without semantic contents}

Now, let us consider that part of the mind consisting in cognitive states -i.e., states which, in one or another way, are related with knowledge. I want to argue that it is not possible a non-regressive and non-circular explanation of the knowledge of the world based on semantic contents without appealing to knowledge of the world which is not based on semantic contents. Moreover, I want to argue that only if we can know directly the world, or to have some kind of direct epistemic access to it, it is possible to know the world through the propositionally articulated semantic contents conveyed by informational processes.

In order to be more precise, we can define "knowledge based on semantic contents" as a knowledge with the structure "I know that a is $\mathrm{F}$ ". In that case, what is known would be that $a$ is $F$. This is the semantic content of my knowledge. Some examples would be "I know that Madrid is the capital of Spain", "I know that Ulysses came back to Ithaca", "I know that passion blinds reason", etc. By "knowledge not based on semantic contents", we can understand a knowledge with the structure "I know a". Here, what is known is not that a has certain feature, or that $a$ is in a certain way. What is known is a. Moreover, in a crucial sense it is known directly. It is not known by means of any sort of propositionally articulated semantic contents. The content of my knowledge, surely partial and tentative, is a itself. Some examples would be "I know Madrid", "I know Ulysses", "I know passion", etc.

With independence of the concepts I have, of the languages I use, of the names or descriptions which make me able to recognize $a$, of the amount of information that I have 
about a, etc., if I know a, then I know the real thing, and I know it directly. I know without intermediaries the authentic a. If I know a, then it could not be other way. As it was noted, this does not mean that I know completely $\mathrm{a}$, in all their aspects, or that $\mathrm{I}$ know a once and for ever. It only means that what I know is the authentic a. In other words, when I know a, I do not know merely some propositionally encapsulated information about a. I know directly the very a

As I said, what I want to argue is that it cannot be explained how we can know the world through contents with the structure that $\mathrm{a}$ is $\mathrm{F}$ without assuming that we also have a kind of direct knowledge of the world, or a kind of direct epistemic access to it, which is not based on such propositionally articulated semantic contents. And the argument is very simple and intuitive. To know that Madrid is the capital of Spain, for instance, could not increase my knowledge of the world unless that, in one or another way, I am able of knowing which part of the world is such Madrid which is the capital of Spain. And I only can be able of that second kind of knowledge, without regress or circularity, knowing Madrid in a direct and non propositional way, or knowing in a direct and non propositional way something from which I can infer that I know which part of the world is Madrid. Knowing more features about Madrid, knowing more propositions about Madrid, would not be enough. Ever it would not be enough to know everything that can be true about Madrid. In order to know which part of the reality is such Madrid that is the capital of Spain, I have to know Madrid in a way that goes beyond any propositional semantic contents of the sort that $a$ is $F$.

The world cannot consist only in information. The world itself has to be constituted by other features apart from informational features. Now, is the world that can be accessible to us something constituted only by semantic contents of a propositional sort? In other words, cannot we know the world but through certain semantic contents like that $a$ is $F$ ? What I am suggesting is that we could not know the world through any amount of semantic contents unless we can also know some of those non informational features of the world in a direct way, i.e., in a way not based on propositionally articulated semantic contents. ${ }^{8}$

\section{Might the world be no more than information?}

That it is not possible to know the world through propositional semantic contents without knowing some features of the world that are not encapsulated that way entails that there is more than information in the world that is epistemically accessible to us --and a fortiori in the world itself. And it entails that our minds cannot be only information.

\subsection{What else can the mind be apart from information?}

Even if the world cannot be only information, the mind might be only information. However, if the mind has to have some kind of direct access to non informational features of the world, then the mind cannot be only information.

According to what we have said, the mind has to have some kind of direct epistemic access to non informational features of the world. That is, the access to such features has to be carried out with independence on any information which can be received, codified and transmitted through semantic contents propositionally articulated. Hence, the world cannot be for the mind merely a "source of quantities of information". And it cannot be merely either a "source of semantic contents". In one or another way, our minds have to be able of being in "close touch" with reality.

What else can be the mind? What else can be the mind apart from quantities of information and apart from a certain number of propositionally articulated semantic contents? A very classical answer to these questions would be that, apart from quantities of information and apart from semantic

8 Russell's distinction between knowledge by description and knowledge by acquaintance, a central element of his philosophy after the formulation of his theory of description, would be very close to our argument. And we can find the same idea in Peirce's discussion of the crucial importance of signs with an indexical character in order to anchor in the real world any other kind of sign. 
contents propositionally articulated, the material structure and composition of the mind enables it to have certain "referential capacities". Referential capacities would offer an epistemic access to the world, even a certain kind of direct knowledge, not based on propositional semantic contents. The important point is that knowing the reference of a propositionally articulated semantic content, knowing how it is anchored in the real world, would not be something like knowing that $a$ is $F$, for any $a$ and $F$. It would be something like knowing directly, without intermediaries of any kind, the very $a^{9}{ }^{9}$

Here, we would have a knowledge not based on semantic contents. And perhaps such sort of knowledge could be of help in order to solve the two problems above noted: the problem of getting selective semantic contents and the problem of error. Let us see why. Referential capacities might be able to select a relevant semantic content from all the other semantic contents which could be also present. That $a$ is $F$, for example, could become in that way selected simply because the system in which that semantic content is exemplified intends to refer to the possibility that the object $a$ is $F$. And because, in principle, those referential capacities could be exercised in a right or wrong way, it might be also possible to exemplify erroneously a semantic content like that $a$ is $F$. This could be so either because we attribute the being $F$ to something that is not $a$, or because we attribute the being $F$ to something that is a but not $F$.

Referential capacities offer a way to try to solve the problems we are focusing on. However, there are reasons to think that it is a wrong way. There are reasons to think that the appeal to referential capacities only presupposes that those problems have a solution. The main difficulty with the assumption of such referential capacities for the mind is that those referential capacities are in serious need of explanation. In no way they would not have to be taken as irreducible. In other words, referential

\footnotetext{
${ }^{9}$ John Searle would be paradigmatic with respect to such appeal to referencial capacities. See in particular Searle, J. (1980, 1983 and 1992).
}

capacities are part of the problem. And because of that, they cannot be the solution.

In fact, many authors have tried not to assume as primitive those referential capacities of the mind. There would be in our minds something more than information. But it would not be a primitive referential capacity. Referential capacities would have to be explained by non-informational features of the mind and, in the end, by non-informational features of the world.

\subsection{Functions}

There are other ways of trying to answer the question about what else can be the mind apart from information. Many recent authors, including here Dretske himself, would say that that "something else" that is not constituted by quantities of information, that does not consist either in some semantic contents propositionally articulated, that "something else" able of selecting particular semantic contents, and also able of making room for the error, is a number of "functions" that the mind has given both its material composition and its peculiar natural history. ${ }^{10}$

Nowadays, a very common perspective in order to explain how the mind is able of selecting determinate semantic contents, semantic contents which also can be erroneously exemplified, appeals to notions such as "functions", "objectives", "control mechanisms", etc. That perspective may have a biological motivation, but it also can be adopted in relation to non biological systems. It could be applied to any system able of receiving and modifying information.

The crucial idea is that not all propositionally articulated semantic content consisting in that $a$ is $F$, that can be transmitted by a signal and received by a certain receiver system, is equally relevant for the system --for its existence, for its efficient functioning, etc.-- And the specific semantic content of a signal could be selected in relation to that "differential relevance". It will be a selected semantic content only that semantic content having a relevant functional role in the informational structure of the

${ }^{10}$ See again Dretske, F. (1981) and Millikan, R. (1984). 
system, with respect to the satisfaction of some objectives associated to the control parameters of determinate processes. Having epistemic access to those functions, objectives, control parameters, etc., in a conscious or unconscious way, the mind would be able to directly access to some features of the world without relying on any semantic content of a propositional kind.

All of that would make us able of giving an answer to the problem of selection. And we could also face the problem of error. The function of having a determinate semantic content, but not others, would be obviously selective. And the possibility that there exists such a function without a correct realization -as when, for instance, some scissors do not serve us to cut something they "would have" to cut-- entails the possibility of exemplifying a determinate semantic content, functionally selected, without being a correct semantic content. Simply, there would be a function which does not have an adequate realization.

From that perspective it is very easy to introduce the biological notion of information. We would be faced with information not only in a quantitative sense, but with information described through particular semantic contents propositionally articulated. Those contents would be functionally selected. And natural selection would be the best candidate -in the domain of non artificial design-- in order to make such functional selections. In that way, we could say, for instance, that some movements in the tail of certain animals have the "function" of transmitting information about a certain danger --predators in the proximities, etc.--. Or that some colours, or gestures, have the "function" of indicating a good disposition for reproduction. Or that certain genetic information has the "function" of controlling the production of determinate proteins, or the production of determinate organs, etc., and that other genetic information is redundant, or simply residual. ${ }^{11}$

There is a vast literature in biology, and philosophy of biology, about these topics. At the more reflective levels, however, an important problem arises. Basically, there are

\footnotetext{
${ }^{11}$ Following that route, some authors have tried to introduce much more sophisticated "functions" like the ones attributed to the "memes".
}

two ways to understand functions. Functions can be understood 1) in a historic and diachronic sense, mainly in relation to the notion of "adaptation", and 2) in a systematic and synchronic sense, connected with the sense in which, for instance, the different parts of a machine can be identified. Indeed, they are two very different ways of making sense of functions. And as it has been noted many times, the implications of those two senses are very different. In particular, those two different senses might entail quite different conclusions with respect to the semantic contents relevantly selected in a particular case.

There would be another important problem. Functions, understood in one or another sense, also would be second order properties. Moreover, in this case they will clearly be functional properties. But the status of functions, in general, as physical properties would be much more problematic than the status of information. Functional properties beyond informational properties have a serious danger of being excluded from the physical world. The problem is that information seems to be a much more basic property than (other) functional properties. In other words, strictly the physical world seems to be constituted by first order physical properties --and relations-- and, may be, by information. And functions would have to be reducible to, or be definable through, those ingredients.

Functions would have to be explained in quite a similar sense to the sense in which it was said that referential capacities would have to be explained. It is very difficult to assume that functions can belong to the physical world as some kind of primitive properties. And this would also apply to what, from a biological point of view, can be called "natural functions". In that respect, the free appeal to the adaptative value of biological functions is very often considered no more than "bad teleology", or mere "panglossianism". In sum, the language of functions seems to have only a heuristic and pedagogic value. 
This would not be to have no value at all, but it would be very different from having a clear and robust ontological value.

\section{Semantic contents from other perspective}

As we have seen, the notion of "function" has not less problems than the notion of "referential capacities". And both notions are much more problematic than the notion of information. Is there some other way of trying to select semantic contents? In this section, I am going to present a very suggesting alternative.

\subsection{Signals and signalizing systems}

Let us think on a signalizing process as something with the following structure:

1) There is a set of particular and concrete objects $O_{1}, O_{2}, \ldots, O_{n}$.

2) There is also another set of particular and concrete objects that serve for signalizing, or marking, the objects mentioned in 1 . We can call them "signals": $s_{1}, s_{2}, \ldots, s_{n}$.

3) An object $o_{i}$ can be signalized always with the signal $s_{j}$, or with different signals $s_{i}, s_{j}$, $\ldots, s_{k}$, in different times and circumstances.

4) The signal $s_{i}$ can always signalize an object $o_{j}$, or different objects $o_{i}, o_{j}, \ldots, o_{k}$, in different times and circumstances.

A signalizing system will be whatever system able of being involved in a signalizing process as the previously described, a very simple process consisting in "registering marks" or in "putting marks".

It would be very important to distinguish between tokens and types of both objects and signals. This would apply especially to conditions 3 and 4 . Strictly, those conditions would have to be expressed in terms of tokens of certain types of objects and signals. Tokens of objects and tokens of signals would belong to certain types. We will assume that there are tokens of objects of types $o_{i}, o_{i}, \ldots$, $o_{k}$, and also that there are tokens of signals of types $s_{1}, s_{2}, \ldots, s_{n}$. For the purposes of our discussion, we will only explicitly distinguish between tokens and types, of objects and properties, when it is necessary.

Now, a semantic content consisting in that $a$ is $F$ could be understood as a property adscribed to certain peculiar combinations of the signals, or marks, that a signalizing system is able of producing. In order to present my proposal, I need first to introduce some other concepts.

\subsection{Correctness conditions}

Let be $R\left(s_{i}, s_{j}\right)$ a particular combination of the signals $s_{i}$ and $s_{j}$ produced by a signalizing system. Let us define $S\left(s_{i}\right)$ as the set of objects signalized by the signal $s_{i}$, and $S\left(s_{j}\right)$ as the set of objects signalized by the signal $s_{i}$. We can establish the following general sort of correctness conditions $\mathrm{C1}$ upon the combination $R\left(s_{i,}, s_{j}\right)$ of signals $s_{i}$ and $s_{j}$ :

(C1) $R\left(s_{i,}, s_{j}\right)$ is a correct combination iff $S\left(s_{i}\right) \subseteq S\left(s_{j}\right)$.

In other words, $R\left(s_{i}, s_{j}\right)$ is a correct combination if, and only if, the objects signalized by $\mathrm{s}_{\mathrm{i}}$ also are objects signalized by $s_{j}$.

\subsection{Condition of re-identification. Signals with a re-identificative value}

Now, let us suppose a complex signalizing system, able of signalizing and also able of producing combinations of signals $R\left(s_{i,}, s_{j}\right)$. Over those combinations of signals, we can establish the following condition of reidentification C2:

(C2) If there are signals $F^{\prime}, F^{\prime \prime}$, etc., such that 1) the system can produce the combinations $R\left(a, F^{\prime}\right), R\left(a, F^{\prime \prime}\right)$, etc., and 2) these combinations of signals satisfy correctness conditions like $C 1$, then we will say that the system is able of reidentifying the objects signalized by $a$.

In that case, we will also say that the signal a becomes a signal with a re-identificative value.

Signals with a re-identificative value are able of signalizing objects in a way that makes possible that those very same objects can be 
signalized by other different signals. Therefore, the same objects could be reidentified through those other signals.

A limit case of C2 would follow when signals $a$ and $F^{\prime}, F^{\prime \prime}$, etc., are tokens of exactly the same relevant type of signal. Here, those different tokens would serve to identify a relevant type of object which is tokened in various ways. From this point of view, every identification would be a re-identification.

Note that the signal $F$ has not been involved in the definition of the condition of reidentification for the signal $a$. This fact will be important in our definition of semantic content.

\subsection{Condition of generalization. Signals with a generalizing value}

Also, we can establish the following condition of generalization C3:

(C3) If there are signals a', a", etc., such that 1) the system can produce the combinations $R(a$ ', $F), R(a$ ", $F)$, etc., and 2) these combinations of signals satisfy some correctness conditions like C1, then we will say that the system is able of generalizing over the objects signalized by $F$.

In that case, we will also say that the signal $F$ becomes a signal with a generalizing value.

From conditions $C 2$ and $C 3$ a number of other important semantic concepts could be defined in new and suggesting ways. We will not do it in this paper. All of them would be based on very simple signalizing processes of the kind above described.

Note that, this time, the signal a has not been involved in the definition of the condition of generalization for the signal $F$. This fact will be also important in our definition of semantic content.

\subsection{Semantic contents}

With the help of the concepts previously introduced, the notion of semantic content could be understood in the following way:
If a system

1) is able of signalizing, i.e., if it is able of registering or putting signals, in the way above described,

2 ) is able of producing the combination of signals, or the complex signal, $R(a, F)$,

3) with respect to other combinations of signals, that is, exceptuating $R(a, F)$, the signal $a$ has for the system a reidentificative value, and

4) with respect to other combinations of signals, that is, exceptuating $R(a, F)$, the signal $F$ has for the system a generalizing value.

then

when the system produces the combination of signals $R(a, F)$, such combination, for the system and in that production, will have the propositionally articulated semantic content consisting in that $a$ is $F$, with the correctness condition associated that $S(a) \subseteq S(F)$.

In other words, a certain combination of signals $R(a, F)$ will have the semantic content that $a$ is $F$ when a signal $a$ with a reidentificative value is combined with a signal $F$ with a generalizing value having that combination $S(a, \subseteq S(F)$ as its correctness condition.

We can say that in those circumstances, that correctness condition makes that the system is committed to signalize with $F$ the objects that are signalized with $a$. And we can say that such correctness condition would be fulfilled, or that it would be satisfied, if the signalizing behaviour of the system shows that in fact this is what the system would probably do in the circumstances.

Some times this will be the case. Other times, it will not. In particular, the last would happen when the system becomes strongly committed with other signalization processes contrary to the previous one -i.e., when the system produces relevant combinations of signals with a content entailing that $a$ is not $F^{12}$

${ }^{12}$ In this respect, correctness conditions would be quite similar to assertability conditions. However, the relevant facts for the acquisition and fulfilment of correctness conditions, in the defined sense, would have 
Hence, the semantic content of a combination of signals such as $R(a, F)$ is crucially determined by

1) the fact that, in the system in question, the signal $a$ has a re-identificative value,

2) the fact that the signal $F$ has a generalizing value, and

3) the fact that the combination of signals $R(a, F)$ has as its correctness condition that $S(a, \subseteq S(F)$.

The re-identificative and generalizing values would be obtained through the satisfaction of conditions $C 2$ and $C 3$ by other combinations distinct from $R(a, F)$. And our definition of semantic content would be applied over the combination of signals $R(a, F)$.

At this point, we can see the reason why the signal $F$ has not been involved in the definition of the re-identificative value of the signal $a$, and also the reason why the signal a has not been involved in the definition of the generalizing value of the signal $F$. In order to have the semantic content that $a$ is $F$, the combination of signals $R(a, F)$ has to have a certain correctness condition such that the signal $a$ has a re-identificative value independent on the satisfaction of that correctness condition. And the combination of signals $R(a, F)$ has to have a certain correctness condition such that the signal $F$ has a generalizing value independent on the satisfaction of that correctness condition.

In other words, to have that $a$ is $F$ as an semantic content with $S(a,) \subseteq S(F)$ as its correctness condition has to be something previous to the satisfaction to that peculiar correctness condition.

\subsection{A progressive selection of semantic contents}

The processes of signalization themselves can be very unclear as to what objects are marked by what signals. In principle, the conditions $\mathrm{C} 1, \mathrm{C} 2$ and $\mathrm{C} 3$ could be applied in many different ways. This entails a problem. It would seem that what we only could obtain is

to do with processes of signalization and not with any kind of irreducible epistemic states. a set of potential semantic contents, without being able to select in no circular or regressive ways some particular semantic contents over other ones.

However, things would not be necessarily so if the sets of possible conditions of correction that could be applicable to a signalizing system were able of have a differential explanatory power in relation to the dynamics and structure of the system, especially when the system increases in complexity. In that case, the semantic contents attributed to the system could become more and more specific.

Even though we did not know with complete certainty what object are marked by what signals, some possibilities could have more explanatory power than other ones. Moreover, an important assumption at this point would be that this is what just happens in the majority of cases. More concretely, the hypothesis would be the following one:

When the conditions of correction are part of complex behaviours and complex structures, the problem of attribute them, obtaining that way certain specific semantic contents, tends to have a unique solution.

The dynamics and structure of the signalizing processes, and its growing complexity, would be able of progressively selecting certain particular semantic contents, or at least a set of logical variations of particular semantic contents. Moreover, such semantic contents could be taken erroneously. That would happen simply when their correctness conditions are not fulfilled.

Following the strategy proposed, semantic contents could be selected. The selection would be compatible with the existence of error. It could be made without any regress or circularity. And we would not need any appeal to the existence of irreducible "referential capacities" or to any sort of natural "functions" in the usual sense.

In the last term, correctness conditions and semantic contents are devices for describing certain peculiar modes of behaviour -including here signalizing behaviour--, which could not be easily described other ways. And to say that the problem of attributing correctness conditions and semantic content to a signalizing system tends to have a unique 
solution, when we know more and more about the dynamics and structural complexities of the systems under consideration, is only to say that such knowledge put us on the good track in order to identify and describe the peculiar modes of behaviour we are interested in.

\subsection{Signalization and reference}

We have suggested the hypothesis that more and more specificity in the attribution of correctness conditions and semantic contents can be achieved through the differential explanatory power that those attributions could have in relation to the dynamics and structure of the signalizing systems. That could be so even though "reference" was not determinate.

The notion of reference, applied to signals, has been completely absent of our approach. There was nothing like "the reference of a signal". The relationships among objects and signals -other objects-- were not relations of "reference", or relations of "aboutness". Signals were not "about" some objects.

Our primitive, or more basic, relation was one in which objects have signals -other objects--, either because a system is able of registering signals, or because the system puts certain marks on some objects. Hence, even though reference is something indeterminate, or even though it becomes something ultimately "inexcrutable"13, our correctness conditions could become more and more selective. And that progressive selection could produce finally unique solutions -and logical variations of those solutions.

Perhaps reference is not determined by physical facts. However, that indetermination is perfectly compatible with the determination in the long run of correctness conditions, and therefore of semantic contents, by the physical facts involved in the processes of signalization. The notions of "object" and "signal", or "mark", --other special kind of object-, are not so clear and unambiguous as for entailing that any process of signalization determines the attribution of some particular correctness conditions and

\footnotetext{
${ }^{13}$ In the sense of Quine, W. (1960 and 1969).
}

semantic contents. In general, there would be more than one such attribution that can be made over any process of signalization. But the more of such physical facts get to be involved in the processes of signalization, and the more we would get to know about them, the narrower will be the margins of variability for such correctness conditions and semantic contents. In the end, those margins of variability only have to do with some relations among $S\left(s_{i}\right)$ and $S\left(s_{j}\right)$, and such entities are sets of physical objects -the physical objects signalized by the signals $s_{i}$ and $s_{j}$.

Once again, our approach does not make any appeal to any kind of "referential capacities" of the mind. Reference has not been supposed as a primitive relation in the processes of signalization. Registering signals, or putting signals, is not referring. Because of that, we can describe how signalizing processes can be carried out, and how through those processes some particular semantic contents can be identified, without any need of conceiving reference as something determinate. In other words, semantic content could be determinate even though reference is not determinate. Moreover, even though reference is not determinate, we could have statements describing reference relations having those statements correctness conditions completely determinate. And some of those statements could be acceptable, but not other ones. ${ }^{14}$

\subsection{Signalizing and naming}

We have argued that signalizing would not be the same than referring. But it would not be the same than naming either. Signalizing is a process, or an operation, much more basic than naming.

Signalizing would be something that could be carried out in a completely "private" way. In "the private diary of sensations" -those of each one--, described by Wittgenstein ${ }^{15}$, there is signalization. There are signalizing

${ }^{14}$ This would follow from the compatibilism between the indetermination of references and the determination of semantic contents, and of their associate correctness conditions. About that compatibilism, see the classical arguments of Post, J. (1987).

${ }^{15}$ See Wittgenstein, L. (1953), especially \# 258 and $\# 270$. 
processes in the sense above defined. Moreover, there could be combinations of signals with associate correctness conditions of kind C1. However, there is only this. And the arguments of Wittgenstein would show that there could not be semantic contents because there is no "private way" in which it can be taken for granted the satisfaction of conditions of re-identification $C 2$ and the satisfaction of conditions of generalization $C 3$. And if naming requires satisfying conditions of re-identification $C 2$, as it is plausible to claim, then there could not be either any naming in such a "private diary of sensations".

\subsection{Signalizing functions? Correctness functions?}

Let I have suggested a strategy to select semantic contents in such a way that 1) the problem to select particular semantic contents can be solved, and 2) such semantic contents can be erroneously exemplified.

The selection could take place simply knowing what objects have what signals. And avoiding all the peculiar traditional problems posed by the notion of reference, this could be made in exactly the same ways in which we get to know something about any object. To know more and more about the dynamics and the structure of the signalizing system would tend to offer unique solutions to that selection.

With respect to the problem of error, things are also clear. Semantic contents would have associate correctness conditions, And those correctness conditions could be fulfilled or not fulfilled. Error would be originated when the particular commitment associated with a semantic content is not displayed in behaviour -at least in potential behaviour. In other words, error is originated when there is a correctness condition which is not fulfilled.

As it was said, such selection would not involve any irreducible "referential capacity". And even taking into consideration dynamical and structural aspects of the signalizing systems, it would not involve any problematic notion of "function" either.

Can we avoid completely the appeal to functions? Could not we say that some "functions of signalization" and, also, some "functions of correction" are implicitly involved in our approach? In fact, we can speak in those terms. But the existence of functions in these senses would not be problematic. Functions would not be something irreducible and unexplainable. Functions of signalizations would simply be certain relations among physical objects. Some physical objects would be signalized, or marked, by other physical objects which are called signals. And functions of correction would simply be certain relations defined over those relations of signalization. They would exist because the second ones exist too.

\subsection{Selective attention}

Any signal would have a certain "quantity of information". Moreover, that quantity of information could be filled with "particular semantic contents", propositionally articulated, through processes of signalization, and through processes of production of combinations of signals satisfying conditions C1, C2 y C3. The selection of semantic contents that has been introduced would not be regressive or circular. In the last term, it is the peculiar dynamics and structure of the processes of signalization what make us able of selecting particular semantic contents with their associate correctness conditions.

In all of that, however, we have to presuppose some sort of "direct access" with respect to the objects that are signalized, and also with respect to the signals that signalize them. Could we say something more about this point? I think that we can. We can say something more. Such sort of "direct access" could be given by what is ordinarily call "attention".

To be attentive is a very basic psychological attitude --really, it is a big familly of attitudes. And it is an attitude that requires at a certain point the lack of intermediaries. At this point, to be attentive to an object, which perhaps also is "calling our attention", is to be in touch with it in a very direct way. The intermediaries -using a monitor, a microscopy, some glasses, etc.-only have an instrumental value. In any case, they are not like a "veil".

Here, we could mention the "qualitative contents" of attention in all of its perceptive modes. That is, we could mention the possible existence of "qualia". And how those "qualia" 
might be infradetermined by any sort of semantic contents propositionally articulated ${ }^{16}$. Even we could make reference to the interesting hypothesis that perhaps semantic contents are constituted by a special sort of modulation, or "tuning", over those qualitative contents ${ }^{17}$. But we will not do it. We can avoid those discussions here. For our purposes, it will be enough to define selective attention in the following way:

To be attentive is to register signals or to put signals.

The proposal is as simple as suggesting. A signalizing system would be a system able to register signal, or able to put signals. In both cases, we could say that the system has a certain sort of selective attention with respect to some objects. And in both cases, we could have first order physical processes supporting informational processes. Those first order physical processed would be able of selecting the particular semantic contents conveyed by the informational processes.

Physical processes consisting in registering signals or putting signals, together with other physical processes consisting in combining signals, could make us to understand how information gets to have the particular semantic contents that it has. Using again the analogy introduced in other sections, we could say that those processes would make us to understand how a glass with the capacity to contain, for instance, $1 / 2$ litre "of some liquid" get to contain in fact $1 / 2$ litre "of water" instead of $1 / 2$ litre "of milk", or instead of $1 / 2$ litre "of beer", etc.

A signalizing system would be a system able to be attentive to reality in a selective way. And this would not be an informational property. It would be something much more basic. In qualitative terms, we could say that a signalizing system would be able of "feeling" and "touching" the world. Of course, these would not be informational properties. And it would be a very suggesting problem to investigate whether selective attention in that

\footnotetext{
${ }^{16}$ About that, see Chalmers, D. (1996) and Tye, M. (1995).

${ }^{17}$ In relation with this idea, see Horgan, T., and J. Tienson (2002), and Pitt, D. (2004).
}

qualitative sense could be also a physical property.

\section{Is there some relevant connection between our two questions?}

Is there some relevant connection between the question whether the world might be only information and the question whether the mind might be only information? We have given a negative answer to both questions. The world cannot be only information, and the mind cannot be only information either. And in fact there is a close and important connection between our answers. The world has to contain non informational features epistemically accessible to us. And our minds have to include something making possible such access.

\subsection{Accessibility and access}

On the one side, knowing the world cannot consist only in knowing quatities of information. It is required to know also some particular semantic contents. And to select some semantic contents entails that some non informational physical features of the world -properties, objects, events, states of affairs, etc.- involved in the informational processes have been accessible. There is no other way of selecting semantic contents. Those features have to exist in reality and they have to be accessible to us. In terms of states of knowledge, we could say that without the appeal to some sort of knowledge not based on semantic contents, propositionally articulated, we could not go out of the situations of regress and circularity to which we are lead by the problems we have faced -mainly, the problem of selecting semantic contents and the problem of error--. And that knowledge not based on semantic contents is knowledge of non informational physical features.

On the other side, if we do not want to appeal to the existence of some sort of primitive "referential capacities", nor to the usual notion of "function", in order to explain how our mind in able to select certain particular semantic contents, i.e., certain particular propositions, we would have to make reference to the special "direct access" 
that our mind has to some of those non informational physical properties.

Mi proposal is that, once we have excluded those "referential capacities" and those "functions", a system -we ourselves, or any signalizing system with a certain degree of complexity- gets to have particular semantic contents registering signals of, or putting signals on, something non informational, and doing it in special ways.

More precisely, only in that way a system can get to have semantic contents progressively determinable. For there is not any reason to think that semantic contents propositionally articulated ought to be always fully determinate!

\subsection{The real world}

Let us close considering a possibility that we have not taking into account. The approach offered about signalizing processes, combinations of signals with characteristic correctness conditions, complexity, etc., would follow having application in a possible world in which every object -event, state of affairs, etc.-is no more than a subjective construction, or projection, with respect to which we are able of maintaining attitudes of selective attention. In other words, it would have application in a completely idealist world. Also, it would have application in a kind of Kantian world bifurcated in a "phenomenal" part and in a "noumenical" part. How to make sense of these possibilities?

I think that the right way of making sense of those possibilities is saying that our discussion is orthogonal to the problem of realism -to "that" problem of realism--. Our discussion might be framed inside a realist conception, but also inside an idealist one. Where does that neutrality come from? I think that the answer is very simple. Knowing the reality does not entail knowing it in all its aspects. And those aspects of reality able of deciding the problem of realism -in the case "that" problem of realism is decidable - are not the aspects of reality able of deciding our discussion about how some particular semantic contents can be obtained!

\section{References}

Chalmers, D. (1996). The Conscious Mind, Oxford: Oxford Univ. Press.

Dretske, F. (1981). Knowledge and the Flow of Information, Cambridge: MIT Press.

Fodor, F. (1990). A theory of Content, Cambridge: MIT Press.

Horgan, T. \& Tienson, J. (2002). The Intentionality of Phenomenology and the Phenomenology of Intentionality. In D.

Chalmers (Ed.) Philosophy of Mind: Classical and Contemporary Readings (pp.520-531). Oxford: Oxford Univ. Press.

Millikan, R. (1984). Language, Thought and Other Biological Cathegories, Cambridge: MIT Press.

Pitt, D. (2004). The Phenomenology of Cognition or What is it Like to Think that P? Philosophy and Phenomenological Research, 69, 1-36.

Post, J. (1987). The Faces of Existence, New York: Cornell Univ. Press.

Quine, W. (1960). Word and Object, Cambridge: MIT Press.

- (1969). Ontological Relativity and Other Essays, New York: Columbia Univ. Press.

Searle, J. (1980). Minds, Brains, and Programs. Behavioral and Brain Sciences, 3, 417-424.

_ (1983). Intentionality: An Essay in the Philosophy of Mind, Cambridge: Cambridge Univ. Press.

- (1992). The Rediscovery of the Mind, Cambridge: MIT Press.

Tye, M. (1995). Ten Problems of Consciousness, Cambridge: MIT Press.

Wittgenstein, L. (1953). Philosophical Investigations, Oxford: Basil Blackwell. 


\begin{abstract}
About the Author
Manuel Liz

Professor of Philosophy (Logic and Philosophy of Science) in the Faculty of Philosophy at the University of La Laguna (Spain). He has been Associated Editor of the Internet philosophical journal Sorites and Secretary of the Spanish philosophical journal Laguna. Member of the Spanish Association for Analytical Philosophy, of the Spanich Society of Logic and Philosophy os Science and of the System Dynamics Society. His research interests are very broad: philosophy of mind, epistemology, metaphysics, philosophy of language, theory of action, philosophy of technology, modeling and simulation, complexity, bounded Rationality, etc. He is author of several books, and chapters of books, in Spanish about topics of those areas. His recent papers include "New Physical Properties" (in Tian Yu Cao (ed.), Philosophy of Science. Proceedings of the Twentieth World Congress of Philosophy, Cambridge, Boston Univ. Press, 2000), "Intentional States: Individuation, Explanation and Supervenience" (in M. José Frápolli \& Esther Romero (eds.), Meaning, Basic Self-Knowledge, and Mind. Essays on Tyler Burge, Stanford, CSLI Lecture Notes, no 132, 2003), "Subjective Experience and External World" (in K. Korta \& J. M. Larrazabal (eds.), Truth, Rationality, Cognition, and Music, Dordrecht, Kluwer (Philosophical Studies Series102, 2004). In colaboration with J. Aracil and M. Vázquez, it would be relevant to mention "An Epistemological Framework for Sustem Dynamics Modelling" (Revue Internationale de Systémique, vol. 9, N1 5, 1995) and "Knowledge and Reality: Some Conceptual Issues in System Dynamics Modelling" (The System Dynamics Review, vol 12, 1996).
\end{abstract}

